\title{
Factors Associated with Depression Score in People with Chronic Obstructive Pulmonary Disease at the North Sumatera University Hospital
}

\author{
Huzaipah Huzaipah (D), Elmeida Effendy*(D), Nazli Mahdinasari Nasution (D) \\ Department of Psychiatry, Faculty of Medicine, Universitas Sumatera Utara, Medan, Indonesia
}

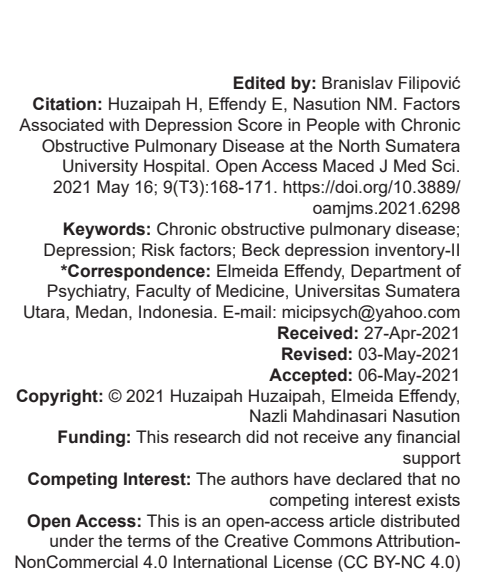

\section{Introduction}

Chronic obstructive pulmonary disease (COPD) is an irreversible lung disease characterized by complaints of dyspnea, chronic cough, and sputum production. One of the leading causes of death and morbidity worldwide is COPD and by 2030 it is estimated that COPD is the third leading cause of death globally [1]. COPD is a progressive disease with an estimated prevalence of 5-13\% [2]. People with COPD experience many comorbidities, of which depression is the most common cooccurring disease in COPD. Estimates of the prevalence of depressive symptoms in people with COPD vary widely, with international estimates ranging from $8 \%$ to $88 \%$ [1], [3]. Between COPD patient and the general population, the risk of developing depression was almost 2-fold higher in COPD patient and a poor prognosis outcome in COPD patient was associated with depression [4], [5], [6]. Feelings of hopelessness, social isolation, reduced physical functioning, and a sedentary lifestyle can be caused by COPD, the increase in depressive symptoms in COPD patient was associated with all of these symptoms. In people with COPD, there are other factors that can also increase the risk of depression it was lack of social support. For reducing symptoms of depression in people with COPD it is important to have high-quality support [6]. Depression in people with COPD leads to more use and health care, and then, it can return people with COPD to the emergency room so that it has an increased economic burden [2].

Isolated and unable to do many social activities due to physical disturbances in people with COPD often occur. When compared with other chronic illnesses, it is not surprising that depression is very common in people with COPD [3]. Depressive symptoms may overlap with COPD symptoms, in clinical practice, these psychiatric problems often go undiagnosed and untreated [3], [6]. In contrast, in people with clinically stable outpatient COPD, the prevalence of major depression (requiring medical intervention) ranges from $19 \%$ to $42 \%$. For determining a diagnosis of depression, due to differences in methodology and variability of the screening questionnaires in the cutoff points so there is no standard approach to the diagnosis of depression in people with COPD. For individuals with COPD, some simple measuring instruments can aid clinical screening for depression and if in doubt, referral to an appropriate specialist can be made, referral to an appropriate specialist has a significant beneficial outcome [3]. 
COPD can lead to a risk of developing depression, the relationship between mood disorders, especially depression and COPD, is bidirectional, which means that mood disorders negatively impact the prognosis of COPD as shown by recent evidence [6]. It is unknown and tends to be multifactorial for the mechanism of the development of depression in people with COPD [3], [6]. Evidence suggests that depression experienced by people with COPD is not a homogeneous entity. In respiratory disease, the presence of environmental factors and the possibility of a genetic predisposition are chronological for the emergence of depression and the neuropsychiatric presence of chronic respiratory disease is an immediate consequence [7].

Therefore, screening and determine the risk factors in people with COPD are essential. This study aims to determine the factors associated with depression score in people with COPD.

\section{Methods}

\section{Patient sample}

This was a multivariate type of predictive study with cross-sectional approach conducted on 119 people with COPD referred to the pulmonology Clinic of North Sumatera University Hospital in Medan, Indonesia, from July 2020 to September 2020. The inclusion criteria were the stable COPD diagnosed confirmed by a single pulmonary diseases subspecialist, participant ages are older than 40 years old and patient signed a written informed consent for the study. The exclusion criteria have had a history of psychiatric disorders, have first degree relatives who have a history of depression, and have a history of substance use (except nicotine and caffeine). The study was confirmed by the ethics committee of the Medicine Faculty of North Sumatera University (decision no: 245/KEP/USU/2020).

\section{Measurement}

Subjects who meet the inclusion and exclusion criteria were given informed consent and were asked to sign the informed consent. After that, anamnesis and structured interviews were carried out to the research subjects, then the subjects were asked to fill biodata and the Beck depression inventory-II (BDI-II) questionnaire for depression screening. After that, the research data were collected and interpreted and processed further. The total BDI-II items consist of 21 subscale items. The time needed to fill this measuring instrument is $5-10 \mathrm{~min}$ and filling orally for $15 \mathrm{~min}$. Guidelines for interpretation of the BDI-II are 0-13: Minimum, 14-19: Mild depression, 20-28: Moderate depression, and 29-63: Major depression. BDI-II with reported Cronbach's $\alpha$ was 0.92 for outpatients and 0.93 for students. Test-retest correlation BDI-II is 0.93 [8]. In Indonesia, the BDI-II Indonesian version validity test was conducted by Ginting et al. in 2012. From the study results, it was found that the Cronbach's $\alpha$ value for the of BDI-II Indonesian version was 0.90 for healthy participants, 0.87 for coronary heart disease, and 0.91 for people with depression. With the acceptance of construct validity of the BDI-II Indonesian version, the results of this study also show that the Indonesian version of BDI-II can be used as a depression screening instrument in Indonesia [9].

\section{Statistical analysis}

Statistical analyses were performed on SPSS ver.22 software. The Kolmogorov-Smirnov test was used to assess normal distribution of quantitative data. In the bivariate analysis for nominal scale independent variables, if the data are normally distributed, the independent t-test will be used and if the data are not normally distributed, a log test will first be carried out but if it is still not normally distributed an alternative test will be carried out using the Mann-Whitney U-test. For numerical scale - independent variable, Pearson's correlation was used to assess the association between independent variables and the dependent variable BDI-II. If a variable is obtained with $p<0.25$ from the bivariate analysis, we can proceed to the multivariate analysis. In this study, there are 10 independent variables, including six independent variables on a numeric scale and four independent variables with a nominal scale. Nominal scale variables are presented with $\mathrm{n}(\%)$, numeric scale variables that are normally distributed are presented with mean \pm standard deviation, numerical variables not normally distributed it presented with the median value (minimum-maximum).

\section{Results}

Table 1 presents a summary of the sociodemographic characteristics of the overall samples. Of the 119 patients with COPD, 91 (76.5\%) were male. The mean age was $61.09 \pm 7.708$ years. The median (min-max) length of education was 12 (6-16) years. The highest frequency of employment status was 67 (56.3\%) which were unemployed. The highest frequency of marital status was $64(53.8 \%)$ which were married. For the smoking status, 91 (76.5\%) were nonsmokers. The median (min-max) body mass index was 20.20 (18.08-24.88). The median (min-max) monthly family income is 2.5 million ( 1 million-5 million). The mean duration of illness was $9.25 \pm 3.445$ years. The median number of comorbidities was $1(0-3)$. In Table 1 , 
from the result of the bivariate analysis, it showed the age $(p<0.001)$, gender $(p=0.057)$, employment status $(p<0.001)$, marital status $(p=0.098)$, smoking status $(p=0.188)$, monthly family income $(p<0.001)$, duration of illness $(p<0.001)$, and the number of comorbidities $(p<0.001)$. From the results of the bivariate analysis, eight independent variables were found with $p<0.25$, then these eight variables were continued into the multivariate linear regression analysis.

Table 1: Sociodemographic and clinical characteristics of patients

\begin{tabular}{|c|c|c|}
\hline Characteristics & COPD patient $(n=119)$ & $p$-value \\
\hline Age, years & $61.09 \pm 7.708$ & $<0.001^{\mathrm{a}}$ \\
\hline \multicolumn{3}{|l|}{ Gender } \\
\hline Male & $91(76.5 \%)$ & \multirow[t]{2}{*}{$0.057^{b}$} \\
\hline Female & $28(23.5 \%)$ & \\
\hline Length of education, years & $12(6-16)$ & $0.331^{\mathrm{a}}$ \\
\hline \multicolumn{3}{|l|}{ Employment status } \\
\hline Employed & $52(43.7 \%)$ & \multirow[t]{2}{*}{$<0.001^{b}$} \\
\hline Unemployed & $67(56.3 \%)$ & \\
\hline \multicolumn{3}{|l|}{ Marital status } \\
\hline Married & $64(53.8 \%)$ & \multirow[t]{2}{*}{$0.098^{c}$} \\
\hline Unmarried & $55(46.2 \%)$ & \\
\hline \multicolumn{3}{|l|}{ Smoking status } \\
\hline Smoker & $28(23.5 \%)$ & \multirow[t]{2}{*}{$0.188^{\mathrm{b}}$} \\
\hline Non-smoker & $91(76.5 \%)$ & \\
\hline Body mass index & $20.20(18.08-24.88)$ & $0.293^{\mathrm{a}}$ \\
\hline Monthly family income & $2.50(1.00-5.00)$ & $<0.001^{\mathrm{a}}$ \\
\hline Duration of illness, years & $9.25 \pm 3.445$ & $<0.001^{\mathrm{a}}$ \\
\hline Number of comorbidities & $1(0-3)$ & $<0.001^{a}$ \\
\hline
\end{tabular}

From Table 2, it showed that there was a significant association between depression and independent variables duration of illness $(p<0.001)$, gender $(p=0.006)$, employment status $(p<0.001)$, and marital status $(p=0.003)$ in people with COPD.

Table 2: Multivariate linier regression analysis

\begin{tabular}{llll}
\hline Variable & Coefficients correlation & Multivariant $\beta$-regression & $p$-value \\
\hline Constant & & 4.55 & 0.001 \\
Duration of illness & 0.303 & 0.69 & $<0.001$ \\
Gender & 0.163 & 3.01 & 0.006 \\
Employment status & 0.491 & 7.76 & $<0.001$ \\
Marital status & 0.183 & 2.88 & 0.003 \\
\hline Adjusted $R^{2}=61.2 \%$. & & &
\end{tabular}

\section{Discussion}

In this study we found that the variables of the duration of illness, female gender, unemployment and unmarried were associated with depression in people with COPD. The duration of illness is considered to be one of the factors that contribute to depression. The longer a person has COPD, the more severe the depression disorder will be, where prolonged COPD can cause helplessness and can also cause financial burdens [10].

In COPD patients, it was stated that female gender had a significant relationship with the occurrence of depression, while several other studies reported no significant relationship between gender and depression [1], [2], [4], [11], [12], [13]. In this study, female more likely suffer from depression. In general, women who suffer from chronic disease, in this case COPD, have a higher depression score. Evidence from some studies, such as in the COPD cohort study, says that the risk of depression in women is 1.19 times greater than that of men, during illness, two reasons can explain this situation, women are more health conscious than men and women are more likely to seek medical treatment is the first reason then also women are traditionally more submissive, passive, and emotional, which can tacitly increase the risk of depression which is the second reason [4]. Men were more sensitive to external careers and goal-oriented factors whereas women showed more sensitivity to interpersonal relationships which was the result of a study of dizygotic twins regarding the relationship between gender and depression in COPD. The fact that hormonal changes in women, especially at puberty, before menstruation, after pregnancy, and during perimenopause, suggest that hormonal fluctuations in women can trigger depression which, in turn, correlates with an increased prevalence of depression [6], [14].

The present study found that employment status was directly associated with depression. In people with unemployment, a contributing factor to the development of depression is living with financial difficulties, which puts people with COPD at a social disadvantage [11], [12]. There was no significant association between unemployment and risk of depression in other reports of COPD [2].

In this study, we also found that marital status also has a relationship with depression score similar to the study by Zhou et al. [12]. Unmarried directly related to depression in people with COPD, which is related to family and social support. Overall, the greater the level of social support a person with COPD feels, the less likely a person with depression will report symptoms [7].

COPD is a chronic and progressive disease that can increase the burden over time. As elderly population increases, the population of people suffering from COPD will increase. Physiological effects of chronic respiratory disease mediate genetic predisposition, environmental factors such as loss and stress, and direct damage to the brain which is a heterogeneous entity with a possible etiology of depression in COPD. During their lifetime, COPD patients may have experienced multiple exposures that increase the risk of developing depression [7]. It is still unclear the exact mechanism of the relationship between mood disorders and COPD [3], [6]. The relationship between depression and impaired pulmonary function may be partly mediated by chronic inflammation based on the results of studies to date; other COPD comorbidities are associated with systemic inflammation and the association between depressive symptoms and pulmonary function is partly mediated by pro-inflammatory cytokines according to available data [6]. Extensively the last few years have been studied about the role of the immune system in chronic disease pathogenesis. Most of the psychiatric conditions attributed to local modulation of microglia cell activity in the central nervous system. Systemic inflammation and high serum concentrations of inflammatory biomarkers 
interleukin (IL)-1 and tumor necrosis factor-alpha have associated them with depression in particular although the nature of the association has not been clearly defined. We have found elevated levels of IL-2, IL-6 and interferon gamma in people with COPD who are depressed compared to healthy controls, although the implications of these findings are unclear from a recent study [4], [5].

\section{Conclusions}

Our findings support previous findings that there is a significant association between duration of illness, gender, employment status, and marital status with depression in people with COPD. In people with COPD, it is recommended to detect psychiatric morbidity with routine screening test. The presence of depression in people with COPD requires more attention and intervention. Pharmacotherapy and nonpharmacotherapy interventions can be given so that it can prevent and also reduce morbidity and mortality for depression in people with COPD.

\section{Acknowledgment}

The authors thanked participants who volunteered for this study and people who participated in the data collection. The authors declare no potential conflicts of interest in writing this original article.

\section{References}

1. Phan T, Carter O, Waterer G, Chung LP, Hawkins M, Rudd C, et al. Determinants for concomitant anxiety and depression in people living with chronic obstructive pulmonary disease. $\mathrm{J}$ Psychosom Res. 2019;120:60-5.

PMid:30929709

2. Zohal M, Mohammadi M, Shamloo F, Javadi A, Yazdi Z. Prevalence and associated depression risk factor in patients with chronic obstructive pulmonary disease in Qazvin, Iran. Eur J Ther. 2019;25(3):159-63.

3. Barnes PJ, Celli BR. Systemic manifestations and comorbidities of COPD. Eur Respir J. 2009;33(5):1165-85.

PMid: 19407051

4. Tzung YT, Livneh H, Lu MC, Tsai PY, Chen PC, Sung FC Increased risk and related factors of depression among patien COPD: A population-based cohort study. BMC Public Health. 2013;13:976

PMid:24138872

5. Zareifopoulos N, Bellou A, Spiropoulou A, Spiropoulou K Prevalence, contribution to disease burden and management of comorbid depression and anxiety in chronic obstructive pulmonary disease. COPD. 2019;16(5-6):406-17.

PMid:31638445

6. Yohannes Am, Kaplan A, Hanania Nicola A. Anxiety and depression in chronic obstructive pulmonary disease: Recognition and management. J Family Pract Cleve Clin J Med. 2018;85(2 Suppl 1):S11-8.

PMid:29494328

7. Norwood RJ. A review of etiologies of depression in COPD. Int $J$ COPD. 2007;2(4):485-91.

PMid:18268923

8. Smarr KL, Keefer AL. Measure of depression and depressive symptoms. Arthitis Care Res. 2011;63(Suppl 11):S454-66. PMid:22588766

9. Ginting H, Naring G, Van der Veld WM, Srisayekti w, Becker ES. Validating the Beck Depression Inventory-II in Indonesia's general population and coronary heart disease patient. Int J Clin Health Psychol. 2013;13:253-42.

10. Mathan K. Demographic profile of patients with comorbid depression in Copd in a tertiary care center in South India a discriptive study. Int J Sci Res. 2019;8(10):36106.

11. Himani G, Badini A, Nanji K. Depression and its association factors among patien with chronic obstructive pulmonary disease in Karachi, Pakistan. Curesu. 2018;10(7):e2930.

PMid:30197852

12. Zhou X, Li J, Gu W, Wang J, Zhu Y, Zhang G, et al. Prevalence and associated factors of anxiety and depression among patient with chronic obstructive pulmonary disease in eight general hospitals in Jiangsu Province of China: A cross-sectional study. Psychiatry Res. 2017;251:48-53.

PMid:28189078

13. Lou P, Zhu Y, Chen P, Zhang P, Yu J, Zhang N, et al. Prevalence and correlation with depression, anxiety and other feature in outpatient with chronic obstructive pulmonary disease in China: A cross-sectional case control study. BMC Pulm Med. 2012;12:53.

PMid:22958576

14. Albert PR. Why is depression more prevalent in women? Psychiatry Neurosci. 2015;40(4):219-21.

PMid:26107348 